\title{
Irreducible Quantum Dynamical Semigroups
}

\author{
David E. Evans \\ Institute of Mathematics, University of Oslo, Blindern, Oslo 3, Norway
}

\begin{abstract}
We study some irreducible and ergodic properties of quantum dynamical semigroups, and apply our methods to semigroups of Lindblad type.
\end{abstract}

\section{§1. Introduction}

There has been great interest recently in the study of dynamical semigroups in quantum systems as semigroups on operator algebras with certain positivity properties such as complete positivity, see for example $[3,4,6-8,11,12,14]$. Here we study some irreducible and ergodic properties of such semigroups, with particular reference to locally completely positive maps.

In $\S 2$ we prove an unbounded version of Lindblad's result relating dissipations and semigroups of locally completely positive maps on $C^{*}$-algebras. In the third section we introduce a concept of an irreducible process, which is weaker than that considered by Davies [3] in the Schrödinger picture. We show that a dynamical semigroup of locally completely positive maps on a $W^{*}$-algebra is irreducible if and only if the largest $W^{*}$-algebra in the fixed point set is trivial. In $\S 4$ we apply our results to semigroups of completely positive maps of Lindblad type $[3,4,11,12]$, and discuss the relationship of our work with that of [3]. In particular we gain more insight into Davies' result on "doubly stochastic" quantum processes [3, Theorem 19]. Some weaker results in these directions have also been obtained by Spohn for completely positive dynamical semigroups of $N$-level systems by entirely different methods [14].

It is a pleasure to thank E. Størmer for his kind hospitality at the University of Oslo, and for helpful comments with the presentation of the manuscript. The author is also grateful to E. B. Davies for arousing his interest in the problem by introducing him to the work of H. Spohn.

\section{§ 2. Dissipations and Locally Completely Positive Semigroups}

Definition 2.1. A linear map $\Phi$ between $C^{*}$-algebras $\mathscr{A}$ and $\mathscr{B}$ is said to be locally completely positive if it satisfies the Kadison-Schwarz inequality:

$$
\|\Phi\| \Phi\left(a^{*} a\right) \geqq \Phi(a)^{*} \Phi(a) \text { for all } a \text { in } \mathscr{A} \text {. }
$$


Note that our definition is slightly different from that of Størmer [15], and that it was shown in $[1,6]$ that a 2-positive linear map is locally completely positive.

Definition 2.2. Let $L$ be a linear map with domain $\mathscr{D}(L)$ in a $C^{*}$-algebra $\mathscr{A}$. Then $L$ is said to be a dissipation $[9,11,12]$ if the following holds:

(i) $\mathscr{D}(L)$ is a $*$-subalgebra of $\mathscr{A}$.

(ii) $L$ is a ${ }^{*}$-map, i.e. $L(x)^{*}=L\left(x^{*}\right)$, for all $x$ in $\mathscr{D}(L)$.

(iii) $L\left(x^{*} x\right) \geqq L\left(x^{*}\right) x+x^{*} L(x)$ for all $x$ in $\mathscr{D}(L)$.

Dissipations generate locally completely positive semigroups, or more precisely we can show the following:

Theorem 2.3. Let $\left\{e^{t L}: t \geqq 0\right\}$ be a strongly continuous semigroup of *-linear maps on a unital $C^{*}$-algebra $\mathscr{A}$. Suppose $\mathscr{D}(L)$ is a subalgebra of $\mathscr{A}$ which is closed under the square root operation of positive elements, and contains the identity of $\mathscr{A}$. Then the following conditions are equivalent:

(2.4) L is a dissipation which kills the identity.

(2.5) $\left\{e^{t L}: t \geqq 0\right\}$ is a semigroup of locally completely positive maps which preserve the identity.

Proof. Suppose (2.4) holds. Then by [9, Theorem 2] $L$ is dissipative; i.e. given $x$ in $\mathscr{D}(L)$ there is a non-zero $f$ in $\mathscr{A}^{*}$ such that $f(x)=\|f\| \cdot\|x\|$, and $\operatorname{Re} f(L x) \leqq 0$. By semigroup theory, this means that $\left\{e^{t L}: t \geqq 0\right\}$ is a semigroup of contractions. But $e^{t L} 1=1$, hence $e^{t L}$ is positive for all positive $t$. It now follows from [6, Theorem 5] that $e^{t L}$ is locally completely positive for all positive $t$.

Remark 2.6. Suppose $L$ is a dissipation on a $C^{*}$-algebra $\mathscr{A}$. Then $L$ being dissipative [9, Theorem 1] is bounded, and the above theorem in this case was shown by Lindblad [11]. From this we can deduce that if $L$ is a *-linear map on a $C^{*}$-algebra $\mathscr{A}$, then $L$ is a dissipation if and only if $\left\{e^{t L}: t \geqq 0\right\}$ is a norm continuous semigroup of locally completely positive contractions.

\section{§ 3. Reduction of Dynamical Semigroups}

We define a dynamical semigroup $\left\{T_{t}: \mathrm{t} \geqq 0\right\}$ on a $W^{*}$-algebra $\mathscr{A}$ to be a semigroup of weak *-continuous positive linear maps which preserve the identity. It is known [13] that any hereditary $W^{*}$-subalgebra of a $W^{*}$-algebra $\mathscr{A}$ is of the form $p \mathscr{A} p$ where $p$ is a projection in $\mathscr{A}$. We say that the hereditary $W^{*}$-subalgebra $\mathscr{B}$ reduces $T_{t}$ if the semigroup $T_{t}$ leaves $\mathscr{B}$ globally invariant and its restriction to $\mathscr{B}$ is a dynamical semigroup. Note that the hereditary $W^{*}$-subalgebra $p \mathscr{A} p$ reduces $T_{t}$ if and only if $T_{t} p=p$ for all positive $t$. In fact, more can be said, if $T_{t} p=p$ for all positive $t$, then $T_{t}(\operatorname{pxp})=p T_{t}(x) p$ for all positive $t$ and $x$ in $\mathscr{A}$, by Broise (see [16]). A dynamical semigroup is said to be irreducible if it is not reduced by any proper hereditary $W^{*}$ subalgebra.

We note that the argument of [3, Theorem 13], can be used to show the following. If $T_{t}$ is a dynamical semigroup on a $W^{*}$-algebra $\mathscr{A}$ which leaves invariant no proper hereditary [13] *-subalgebra of $\mathscr{A}$, then $T_{t}$ has no non-scalar fixed points i.e. $\left\{x \in \mathscr{A}: T_{t}(x)=x, \forall t\right]=\mathbb{C}$. See also (3.4) of Theorem 3.1 . 
The reduction of processes has also been considered in $[3,5]$, but their definitions differ from ours. In [5] not all hereditary $W^{*}$-subalgebras are considered, in fact only the weakly closed two sided ideals are admissable i.e. $\{p \mathscr{A}: p$ a central projection in $\mathscr{A}\}$. In [3] the author works in the Schrödinger picture, i.e. he considers the semigroup $\left(T_{t}\right)_{*}$ on the predual $\mathscr{A}_{*}$, and his admissable subspaces in $\mathscr{A}_{*}$ are the norm closed hereditary subspaces on $\mathscr{A}_{*}$. If $\mathscr{A}=\mathscr{B}(\mathscr{H})$, these are $\{p \mathscr{T}(\mathscr{H}) p: p$ a projection in $\mathscr{B}(\mathscr{H})\}$. Clearly, if $p$ is a projection in $\mathscr{B}(\mathscr{H})$, then a $W^{*}$ dynamical semigroup $\left\{T_{t}: t \geqq 0\right\}$ leaves $p \mathscr{B}(\mathscr{H}) p$ globally invariant if and only if $\left\{\left(T_{t}\right)_{*}: t \geqq 0\right\}$ leaves $(1-p) T(H)(1-p)$ invariant. However our definition is stronger, since we impose the normalising condition $T_{t} p=p$, for all $t \leqq 0$.

Theorem 3.1. Let $T_{t}$ be a dynamical semigroup of locally completely positive maps on a $W^{*}$-algebra $\mathscr{A}$. Then

(3.2) The set $\left\{x \in A: T_{t}\left(x^{*} x\right)=x^{*} x, T_{t}(x)=x\right\}$ is a weakly closed subalgebra of $\mathscr{A}$.

(3.3) The hereditary $p \mathscr{A} p$ reduces $T_{t}$ if and only if $T_{t}(y p)=T_{t}(y) p$ for all positive $t$, and $y$ in $\mathscr{A}$.

(3.4) $T_{t}$ is irreducible if and only if $\left\{x \in \mathscr{A}: T_{t}\left(x^{*} x\right)=x^{*} x, T_{t}\left(x x^{*}\right)=x x^{*}, T_{t}(x)=x, \forall t\right\}=\mathbb{C}$.

Proof. Fix $t \geqq 0$, and define $D: \mathscr{A} \times \mathscr{A} \rightarrow \mathscr{A}$ by $D(x, y)=T_{t}\left(x^{*} y\right)-T_{t}\left(x^{*}\right) T_{t}(y)$. Then $D$ is sesquilinear, and $D(y, y) \geqq 0$ for all $y$ in $\mathscr{A}$. Thus the Cauchy-Schwarz inequality applied to $\varphi D$, for $\varphi$ in $\mathscr{A}_{+}^{*}$ shows that if $x \in \mathscr{A}$, then $D(x, x)=0$, if and only if $D(x, y)=0$ for all $y$ in $\mathscr{A}$. Thus $T_{t}\left(x^{*} x\right)=x^{*} x$ and $T_{t}(x)=x$ for all $t \geqq 0$, if and only if $T_{t}(y x)=T_{t}(y) x$ for all $y$ in $\mathscr{A}$. The statements of the theorem now clearly follow.

We denote the subalgebra in (3.2) by $\mathscr{A}(T)$.

Remark 3.5. The proof of the above Theorem can be used to strengthen Theorem 3.1 in [1], and thus Corollary 3.2 and Theorem 3.3 of that paper also apply to locally completely positive maps. See also the proof of [2, Theorem 3.1].

\section{§4. Applications}

We are now going to apply the preceeding results to dynamical semigroups of Lindblad type (see $[3,4,7,8,11,12,14])$. Let $(\Omega, \mu)$ be a $\sigma$-finite measure space, $\mathscr{H}$ a separable hilbert space, and $A$ a weakly measurable map from $\Omega$ into $\mathscr{B}(\mathscr{H})$ such that the map $\xi \rightarrow A(\cdot) \xi$ defines a bounded linear operator $\mathfrak{A}$ from $\mathscr{H}$ into $L^{2}(\Omega ; \mathscr{H})$. Then there is a normal completely positive linear map $V$ on $\mathscr{B}(\mathscr{H})$ given by

$$
V(x)=\int_{\Omega} A(\omega)^{*} x A(\omega) d \mu(\omega),
$$

where the integral converges in the ultraweak topology, and $0 \leqq V(1) \leqq\|\mathfrak{A}\|^{2}$. (Note that Kraus [10] has shown that any normal completely positive map on $\mathscr{B}(\mathscr{H})$ arises in this way from an atomic measure on a countable set.) Let $H$ be a bounded self adjoint operator on $\mathscr{H}$ and define

$$
K=i H-\frac{1}{2} V(1) \text {. }
$$


Then $L(x)=V(x)+K^{*} x+x K, x \in \mathscr{B}(\mathscr{H})$ defines a norm continuous semigroup $\left\{e^{t L}: t \geqq 0\right\}$ of completely positive normal maps on $\mathscr{B}(\mathscr{H})$. We can now show the following.

Theorem 4.1. Let $\mathscr{M}$ be a von Neumann algebra on the hilbert space $\mathscr{H}$, which is globally invariant under the semigroup $e^{t L}$. If $T_{t}$ is the induced $W^{*}$-dynamical semigroup on $M$, then the algebra $\mathscr{M}(T) \equiv\left\{x \in M: T_{t}\left(x^{*} x\right)=x^{*} x, T_{t}(x)=x, \forall t \geqq 0\right\}$ is equal to $\mathscr{M} \cap\{A(\omega), K\}^{\prime}$. Thus $T_{t}$ is irreducible if and only if $\mathscr{M} \cap\left\{A(\omega), A(\omega)^{*}, H\right\}^{\prime}=\mathbb{C}$. (Here the commutants are interpreted in an almost everywhere sense.)

Proof. We have the following identity valid for all $x$ in $\mathscr{B}(\mathscr{H})$ :

$$
L\left(x^{*} x\right)-L\left(x^{*}\right) x-x^{*} L(x)=\int_{\Omega}[A(\omega), x]^{*}[A(\omega), x] d \mu(\omega) .
$$

Thus if $x \in \mathscr{M}(T)$, we have $L\left(x^{*} x\right)=L(x)=0$, so that $[A(\omega), x]=0$, a.e. This implies that $V(x)=V(1) x$, so that $0=L(x)=V(x)+K^{*} x+x K$ implies that

$V(1) x+(-i H-V(1) / 2) x+x(i H-V(1) / 2)=0$

i.e. $x(i H-V(1) / 2)=(i H-V(1) / 2) x$

i.e. $[x, K]=0$.

Conversely, if $x \in \mathscr{M} \cap\{A(\omega), K\}^{\prime}$, we have $V(x)=V(1) x$, so that by the above manipulations $L(x)=V(1) x+K^{*} x+x K=0$, and also $L\left(x^{*} x\right)=0$ by (4.2).

It follows easily, and also from [6, Theorem 3$]$ that

$$
\mathscr{M}(T) \cap \mathscr{M}(T)^{*}=\mathscr{M} \cap\left\{A(\omega), A(\omega)^{*}, H\right\}^{\prime} .
$$

Remark 4.3. We briefly discuss the relation of this work with that of [3] when $M=\mathscr{B}(\mathscr{H})$ in Theorem 4.1. Then as noted previously, if the process $\left(T_{t}\right)_{*}$ is irreducible in the sense of [3], then the process $T_{t}$ is also irreducible in our sense. If $\left(T_{t}\right)$, is irreducible, then clearly there is no proper closed subspace of $\mathscr{H}$ invariant under $K$ and $A(\omega)$ (a.e.). If $\Omega$ is a separable locally compact Hausdorff space, and $A$ is strongly continuous, then the converse is also true [3]. Now if $A(\omega)$ is normal almost everywhere then $\{A(\omega), K\}^{\prime}$ is a *-algebra, and equals $\left\{A(\omega), A(\omega)^{*}, H\right\}^{\prime}$ $=\{A(\omega), H\}^{\prime}$. In this "doubly stochastic" situation it was shown in [3, Theorem 19] that if $\left(T_{t}\right)_{*}$ is an irreducible infinite dimensional process, then it has no equilibrium state. Moreover it was shown in [3, Theorem 13] that if $\left(T_{t}\right)_{*}$ is an irreducible process on a finite dimensional system, then it has an unique equilibrium state. In this situation, the doubly stochastic assumption was not required.

Now suppose the dynamical semigroup $T_{t}$ leaves invariant $\varrho$ in $\mathscr{T}(\mathscr{H})$, i.e. $\left(T_{t}\right)_{*} \varrho=\varrho$, for all positive $t$, or equivalently $L_{*}(\varrho)=0$. Clearly, it is enough to consider self adjoint $\varrho$. Then if either the process is doubly stochastic [i.e. $A(\omega)$ is normal a.e.] or $\mathscr{H}$ is finite dimensional with $L_{*}(1)=0$, then :

$$
\begin{aligned}
& L_{*}\left(\theta^{*} \theta\right)-L_{*}\left(\theta^{*}\right) \theta-\theta^{*} L(\theta) \\
& =\int_{\Omega}\left[A(\omega)^{*}, \theta\right]^{*}\left[A(\omega)^{*}, \theta\right] d \mu(\omega)
\end{aligned}
$$

for all $\theta$ in $\mathscr{T}(\mathscr{H})$. But $\operatorname{tr} L_{*}(\theta)=0$, for all $\theta$ in $\mathscr{T}(\mathscr{H})$, hence:

$$
\operatorname{tr}\left[L_{*}\left(\varrho^{2}\right)-L_{*}(\varrho) \varrho-\varrho L_{*}(\varrho)\right]=0 \text {. }
$$


It follows from (4.4) and (4.5) that $\left[A(\omega)^{*}, \varrho\right]=0$, and $\left[K^{*}, \varrho\right]=0$, i.e. $\varrho \in\{A(\omega), K\}^{\prime}$. Thus if the process $T_{t}$ is irreducible, we have that $\varrho \in \mathbb{C}$. In the infinite dimensional case, this means that $\varrho=0$, and there is no equilibrium state, although $\operatorname{tr}$ is an invariant weight. However in finite dimensions we have an unique equilibrium state.

Acknowledgement. We would like to thank the referee for his comments.

\section{References}

1. Choi,M.D.: Ill. J. Math. 18, 565-574 (1974)

2. Choi, M.D., Effros, E.G.: J. Funct. Anal. 24, 156-209 (1977)

3. Davies, E. B.: Commun. math. Phys. 19, 83-105 (1970)

4. Davies, E. B. : Z. Wahrscheinlichkeitsth. 23, 261-273 (1972)

5. Edwards, C. M.: Commun. math. Phys. 16, 207-230 (1970)

6. Evans, D.E.: Commun. math. Phys. 48, 15-22 (1976)

7. Evans, D.E., Lewis,J.T.: Commun. math. Phys. 50, 219-227 (1976)

8. Gorini,V., Frigerio, A., Verri,M., Kossakowski,A., Sudarshan,E.C.G.: Properties of quantum Markovian master equations (preprint IFUM 188/FT, Milan, 1976)

9. Kishimoto, A.: Commun. math. Phys. 47, 25-32 (1976)

10. Kraus, K.: Ann. Phys. 64, 311-335 (1971)

11. Lindblad,G. : Commun. math. Phys. 48, 119-130 (1976)

12. Lindblad,G. : Letters math. Phys. 1, 219-224 (1976)

13. Pedersen,G.K.: An introduction to $C^{*}$-algebra theory (Chapters I, II) (preprint) Copenhagen 1974

14. Spohn,H.: An algebraic condition for the approach to equilibrium of an open $N$-level system (preprint) München 1976

15. Størmer, E.: Acta math. 110, 233-278 (1963)

16. Størmer, E.: Positive linear maps and Jordan homomorphisms of $C^{*}$-algebras. Oslo University (1969)

Communicated by H. Araki

Received November 15, 1976; in revised form March 1, 1977 
